\title{
Simply Supported Biharmonic Equations with Polynomial Data in The Unit Ball of $R^{n}$
}

\author{
Agah D. Garnadi
}

\begin{abstract}
We studied simply supported boundary value problem of Biharmonic equation in the unit ball of $R^{n}, n \geq 3$, with polynomial data. The problem is restated as a pair of Laplace and Poisson equations with polynomial Dirichlet problems. We utilize an exact algorithms for solving Laplace equations with Dirichlet boundary conditions with polynomial functions data. The algorithm requires only differentiation of the boundary data, but no integration.

Keywords: Dirichlet Problem, Harmonic Polynomials, Simply Supported Mathematics Subject Classification : Primary 31B05, 31B20
\end{abstract}

\section{Introduction}

In this paper, we studied Biharmonic equation with simply supported type boundary value problem with polynomials data in $R^{n}$. We approached the solution by Lemma that has been formulated by [2] and Lemma by [3].

Let $x=\left(x_{1}, x_{2}, x_{3}, \cdots, x_{n}\right) \in R^{n}, n \geq$, thus for $\alpha=\left(\alpha_{1}, \alpha_{2}, \alpha_{3}, \cdots, \alpha_{n}\right)$ of nonnegative integers we say $x^{\alpha}$ as monomial $x_{1}^{\alpha_{1}} x_{2}^{\alpha_{2}} x_{3}^{\alpha_{3}} \cdots x_{n}^{\alpha_{n}}$. The degree of $x^{\alpha}$ is $\alpha_{1}+\alpha_{2}+\alpha_{3}+\cdots+\alpha_{n}$. A polynomial is said to be homogeneous of degree $m$ if it is a finite linear combination of monomial $x^{\alpha}$ of degree $m ; m=0,1,2, \ldots \ldots$. Let $\mathcal{P}_{m}$ denotes the vector space of polynomials in $R^{n}$, homogeneous of degree $m$, and $\mathcal{H}_{m}$ is the subspace of harmonic polynomials of degree $m$, then we have property by Axler and Ramey following

$$
\mathcal{P}_{m}=\mathcal{H}_{m}+|x|^{2} \mathcal{P}_{m-2} .
$$

Theorem 1.1 [Axler \& Ramey] If $p \in \mathcal{P}_{m}$, then $p=\Lambda_{m}(p)+|x|^{2} q$, for some $q \in \mathcal{P}_{m-2}$ and $\Lambda_{m}(p) \in \mathcal{H}_{m}$. 
Theorem 1.1 leads to the following corollary, which gives the well-known decomposition (2) and an explicit formula for $p_{m}$.

Corollary 1.2 Every $p \in \mathcal{P}_{m}$ can be uniquely written in the form

$$
p=p_{m}+|x|^{2} p_{m-2}+\ldots+|x|^{2 k} p_{m-2 k},
$$

where $k=\left[\frac{m}{2}\right]$ and $p_{j} \in \mathcal{H}_{j}$ for each $j$. Furthermore, $p_{m}=\Lambda_{m}(0)$.

Let $\Omega$ is the unit ball in $R^{n}$, with the boundary $\Gamma=\partial \Omega=\left\{x \in R^{n} \mid x_{1}^{2}+x_{2}^{2}+\right.$ $\left.x_{3}^{2}+\cdots+x_{n}^{2}=1\right\}$. The Biharmonic equation can be reformulated as following:

$$
\Delta^{2} u=0 \text { in } \Omega
$$

with boundary value problems

$$
\left.u\right|_{\Gamma}=p \text {, and }
$$

$$
\left.\Delta u\right|_{\Gamma}=q,
$$

q where, $p$ and $q$ is polynomials function.

The main result of this paper is to state the Theorem for the solution of problem in (2). The solution of this problem is a polynomial function that given in Theorem 3.1 .

\section{Technical Lemmas}

This following Lemmas is needed to solve the Biharmonic equation:

Lemma 2.1 If $p \in \mathcal{P}_{m}$, then the solution to the Dirichlet problem, $u \in \mathcal{P}_{m}$, with boundary data $\left.u\right|_{\Gamma}=p$ is

$$
p_{m}+p_{m-2}+\ldots+p_{m-2 k}
$$

where $k=\left[\frac{m}{2}\right]$ and $p_{m}, p_{m-2}, \ldots, p_{m-2 k}$ are the harmonic polynomials.

Proof:

Suppose $p \in \mathcal{P}_{m}$, because $|x|=1$ in boundary, by using (1) we have (3) equals to $p$ on $\Gamma$. Obviously (3) is harmonic on $R^{n}$, and hence its restriction to $\Omega$ is the solution to the Dirichlet problem.

Lemma 2.1 said that if we have polynomial data for Dirichlet problem then we can obtain the solution of the Laplace equation with this boundary value problem. The solution is a linear combination of harmonic functions. We recommend you to study the algorithm to obtain this harmonic polynomials in [2]. 
By setting $p_{m-1}, p_{m-3}, \ldots$ are equal to 0 , the solution $u$ can be written as

$$
u=\sum_{j=0}^{m} p_{j}
$$

55

\section{${ }_{73}$ Acknowledgement}

74

where $\left.\sum_{j=0}^{m} p_{j}\right|_{\Gamma}=p$. $u \in \mathcal{P}_{m+2}$ such that

\section{Theorem}

and problem:

Lemma 2.2 [Herzog, 2000] Given $p \in \mathcal{P}_{m}$ and $q \in \mathcal{P}_{m+2}$ then there exists

$$
\Delta u(x)=q(x) \quad x \in \Omega \quad \text { and }\left.\quad u\right|_{\Gamma}=p(x) .
$$

Here we provide the Theorem about the solution of Biharmonic equation. The Existence of this solution can be presented in the following Theorem:

Theorem 3.1 Given $p \in \mathcal{P}_{m}$ and $q \in \mathcal{P}_{m+2}$ then there exists $u_{h} \in \mathcal{P}_{m}$ and $u_{2} \in \mathcal{P}_{m+2}$ such that $u=u_{h}+u_{p}$ is the solution of (2).

Proof: The Biharmonic in (2) can be writen as following:

$$
\Delta u=v \quad ;\left.u\right|_{\Gamma}=p,
$$

$$
\Delta v=0 \quad ;\left.v\right|_{\Gamma}=q .
$$

The solution of problem (4) can be formulated if we had solved the problem (5). The solution of problem (5), $v \in \mathcal{P}_{m+2}$, can be obtained by using Lemma 2.1 which is a harmonic polynomials function.

Suppose that the solution of (4) is $u=u_{h}+u_{p}$, where $u_{h}$ is the solution of this

$$
\Delta u_{h}=0 \quad ;\left.u_{h}\right|_{\Gamma}=p
$$

and $u_{p}$ is the solution of this problem:

$$
\Delta u_{p}=v \quad ;\left.u_{p}\right|_{\Gamma}=0 .
$$

The solution $u_{h}$ can be obtained by using Lemma 2.1 which is the harmonic polynomials function. Next, by solving problem (7) when the solution of this problem is guaranteed by Lemma 2.2, so we have $u_{p}$.

This work partially funded by PUPT-IPB under contract no 083/5122H/PL/Dit.Litabmas/II/2015. 


\section{References}

76 [1] Sheldon Axler, Paul Bourdon, and Wade Ramey, Harmonic Function Theory, Graduate Text in Mathematics, Springer-Verlag, 1992.

[2] S. Axler and W. Ramey, Harmonic polynomials and Dirichlet type problems, proc. Amer. Math. Soc. 123(12) (1995), 3765-3773.

[3] G. Herzog, Polynomials solving Dirichlet boundary value problems, Amer. Math. Mothly 107(10) (2000), 934-936. 